\title{
A review of advances in China's flash flood early-warning system
}

\author{
Changjun Liu ${ }^{1,2}$ (D) Liang Guo ${ }^{1,2} \cdot$ Lei $^{3} e^{3}$ Shunfu Zhang ${ }^{1,2} \cdot$ \\ Yanzeng $\mathrm{Zhao}^{4} \cdot$ Tianyu Song ${ }^{3}$
}

Received: 28 July 2017/ Accepted: 4 January 2018/Published online: 21 April 2018

(C) The Author(s) 2018

\begin{abstract}
This paper summarizes the main flash flood early-warning systems of America, Europe, Japan, and Taiwan China and discusses their advantages and disadvantages. The latest development in flash flood prevention is also presented. China's flash flood prevention system involves three stages. Herein, the warning methods and achievements in the first two stages are introduced in detail. Based on the worldwide experience of flash flood early-warning systems, the general research idea of the third stage is proposed from the viewpoint of requirements for flash flood prevention and construction progress of the next stage in China. Real-time dynamic warning systems can be applied to the early-warning platform at four levels (central level, provincial level, municipal level, and county level) . Through this, soil moisture, peak flow, and water level can be calculated in real-time using distributed hydrological models, and then flash flood warning indexes can be computed based on defined thresholds of runoff and water level. A compound warning index (CWI) can be applied to regions where rainfall and water level are measured by simple equipment. In this manner, flash-flood-related factors such as rainfall intensity and antecedent and cumulative rainfall depths can be determined using the CWI method. The proposed methodology for the third stage could support flash flood prevention measures in the 13th
\end{abstract}

Changjun Liu

1cj2005@iwhr.com

1 Research Center on Flood and Drought Disaster Reduction of the MWR, Beijing 100038, China

2 Research Center on Flood and Drought Disaster Reduction, China Institute of Water Resources and Hydropower Research, Beijing 100038, China

3 School of Hydraulic Engineering, Dalian University of Technology, Dalian 116024, China

4 Hydrology and Water Resources Bureau of Henan Province, Zhengzhou 450003, China 
5-Year Plan for Economic and Social Development of the People's Republic of China (2016-2020). The research achievements will serve as a guidance for flash flood monitoring and warning as well as flood warning in medium and small rivers.

Keywords Flash flood disaster - Early-warning index - Dynamic warning - Antecedent precipitation index $\cdot$ Accumulated precipitation

\section{Introduction}

With changes in the global climate, the frequency of extreme precipitation and flash flood disasters is increasing. As a result, flash flood has become a major natural hazard all over the world (Han et al. 2015; Kvočka et al. 2016). In China, flash floods are characterized by widespread distribution, large quantities, high burstiness, and destructiveness attributed to complex geographic and geomorphic conditions and various climate types. In addition, the forecasting and warning of flash floods in China is faced with various challenges because of the abundant small-scale catchments in mountainous areas with steep topographic slopes, which are easily affected by the microclimate and local climate (Kim and Choi 2015).

China has invested more than 28 billion Renminbi (RMB) in flash flood prevention, involving a total area of 3.86 million $\mathrm{km}^{2}$ distributed over 2058 counties in 30 provinces (autonomous regions and municipalities). China's Ministry of Water Resources has already conducted numerous studies on flash flood disaster control. Over recent decades, national flash flood prevention projects, comprising non-structural and supplementary structural measures, have been implemented in China. The National Flash Flood Control Planning (NFFCP) was compiled during the period of 2003-2006 (Sun et al. 2012), and pilot projects for flash flood prevention were implemented in 103 counties during 2008-2009. The construction of non-structural measures (other than engineering measures) for flash flood prevention was officially initiated in 1836 counties during 2010-2012 (Sun et al. 2012). From 2013 to 2015, national flash flood prevention projects, including the national flash flood hazard investigation and evaluation, and national middle and small rivers and dam reinforcement, entered an overall implementation stage. Several achievements have been made in the national flash flood hazard investigation and evaluation, such as the nationwide investigation of prevention area and inhabitant distribution, systematic analysis of underlying surface conditions and hydrological features in small-scale mountainous catchments, study of storm and flood characteristics, development of a calculation method for early-warning and threshold indexes, and establishment of a national flash flood hazard investigation and evaluation database. In 2016, research on flash flood prevention was conducted considering three aspects: estimating flash floods and checking early-warning indexes, monitoring and analyzing antecedent precipitation, and fine forecasting and warning of flash floods. These three aspects involved the clustering of distributed hydrological models, multi-source rainfall data fusion, vertical-mixed runoff model, channel flood routing model, and reservoir regulation. Through such efforts, the accuracy of the early-warning index has been improved. Chinese scholars are also making great efforts to develop new methods that are truly advanced and appropriate to China's needs of flash flood prevention (He and Li 2014; Hu 2016; Huang et al. 2016; Miao et al. 2016; Yang et al. 2016; Zeng et al. 2016; Wei et al. 2017). China's flash flood prevention has been progressively developed from non-existent to overall scientific decision-making support, from empirical methods to early-warning index systems, and will be improved from the 
monitoring and early-warning platform at the county level to information sharing and construction at the central, provincial, municipal, and county levels. It has been found that the monitoring and early-warning platform has prevented more than 740 thousand people from flash flood disasters. A statistical chart of the number of flash floods in China is shown in Fig. 1 (Guo et al. 2017). Since 2003, the number of flash floods (caused casualties) shows a decline trend with some fluctuations which are due to the extreme rainfall in 2007, 2010, and 2016. Compared with 1950-2002, the number of flash floods (caused casualties) accounts for a small fraction (less than 15\%) of the total number. It indicates China's national flash flood prevention projects have indeed played a significant role in flood control and disaster reduction. Flash flood early-warning systems have significantly contributed to the protection of lives and property not only in China but throughout the world as well (Nieland and Mushtaq 2016).

In general, flash flood early-warning indexes include the precipitation early-warning index and water level early-warning index. The water level early-warning index is usually applied to warning in small or medium-sized basins. However, this index has not been applied in China because the concentration time is extremely short in small-scale mountainous areas and automatic monitoring stations cannot cover all the areas. Therefore, the precipitation early-warning index is introduced in detail in this paper. Based on the summary and analysis of worldwide methods and systems of precipitation early-warning indexes, this paper introduces China's current precipitation early-warning index methods. Considering new technologies and requirements for future flash flood prevention systems for China, a hierarchical early-warning index system and its calculation methods are proposed as theoretical support to the 13th 5-Year Plan for Economic and Social Development of the People's Republic of China (2016-2020).

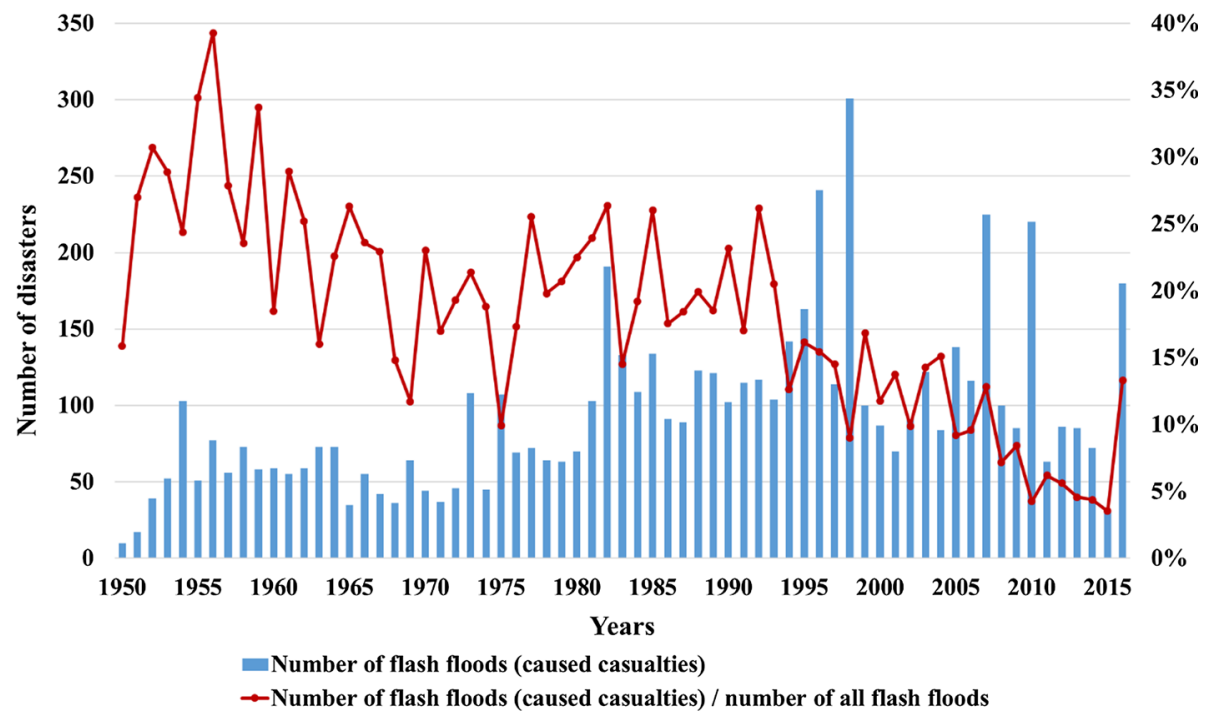

Fig. 1 Number and casualties of flash floods in China 


\section{Research status of flash flood early-warning index}

Over the past decade, worldwide developments have been made to improve flash flood forecasts, including remotely sensed data, flow forecast models and methods, and forecast uncertainty estimates (Hapuarachchi et al. 2011). Gourley et al. (2013) announced an assembled database on flash floods for the entire USA, which is available in multiple, common formats to a wide range of users. Yuan et al. (2017) presented a quality validation approach that uses Anselin Local Moran's I (DM-Moran), which is based on a flash flood model and a spatial data mining algorithm. They used the DM-Moran model to validate the quality of the data from the Chinese Flash Flood Survey and Evaluation (CFFSE) project. Worldwide research on flash flood prevention focuses on the early-warning index, especially rainfall threshold (Golian et al. 2010). Rainfall threshold presents the value of rainfall intensity and accumulated precipitation that occurs immediately prior to flash flood events. This value is related to the characteristic of precipitation, soil moisture, and underlying surface (Diakakis 2011; Yucel 2015). Gourley et al. (2012) evaluated Flash Flood Guidance (FFG) values and developed the gridded FFG used by the National Weather Service (NWS) to monitor and predict imminent flash flooding. Clark et al. (2014) established the benchmark skill of the operational flash flood guidance (FFG) product used by NWS to forecast, monitor, and warn the public about dangerous flash flooding events. Zeng et al. (2016) developed a Cascading Flash Flood Guidance system, progressively from the Flash Flood Potential Index, the Flash Flood Hazard Index to the Flash Flood Risk Index. Compared to other approaches, the CFFG system could be a useful prototype in mapping the characteristics of flash floods in China in a cascading manner. Saito and Matsuyama (2015) produced a preliminary high-resolution probable hourly precipitation $\left(\mathrm{mm} \mathrm{h}^{-1}\right)$ and probable Soil Water Index (SWI). They used a 50-yr recurrence interval over the Japanese archipelago from 5-km grid-cell Radar/Rain Gauge-Analyzed Precipitation (R/A) with a 26-yr time series (1988-2013). Based on precipitation, soil moisture, and underlying surface, significant achievements have been made in previous research, including FFG (Carpenter et al. 1999), SWI (Sugawara et al. 1974), and Rainfall Triggering Index (RTI) (Jan and Lee 2004). America, Europe, Japan, and Taiwan China are the flash flood prone areas, and the research of flash flood early-warning system in these regions could represent the highest level of the world. Therefore, the principles, methods, and advantages of these indexes are summarized and shown in Table 1. Moreover, current early-warning index calculation methods are shown in Table 2. These common methods are widely used in China and around the world and can be classified into three types: statistical induction, hydraulics, and hydrological model.

\section{Causes and characteristics of flash floods in China}

Factors that cause disastrous flash floods are associated with both nature and the society. Flash floods likely form and develop as a consequence of joint contributions of several factors, such as precipitation, topographic and geological conditions, and human activities. Precipitation is a direct factor and trigger of flash floods. Heavy rainfall events, especially strong rainfall intensity in a short duration, have a high likelihood of causing flash floods. Mountainous areas cover more than two-thirds of China's land area, and certain topographic and geological conditions may intensify flash flood damages. Moreover, most 


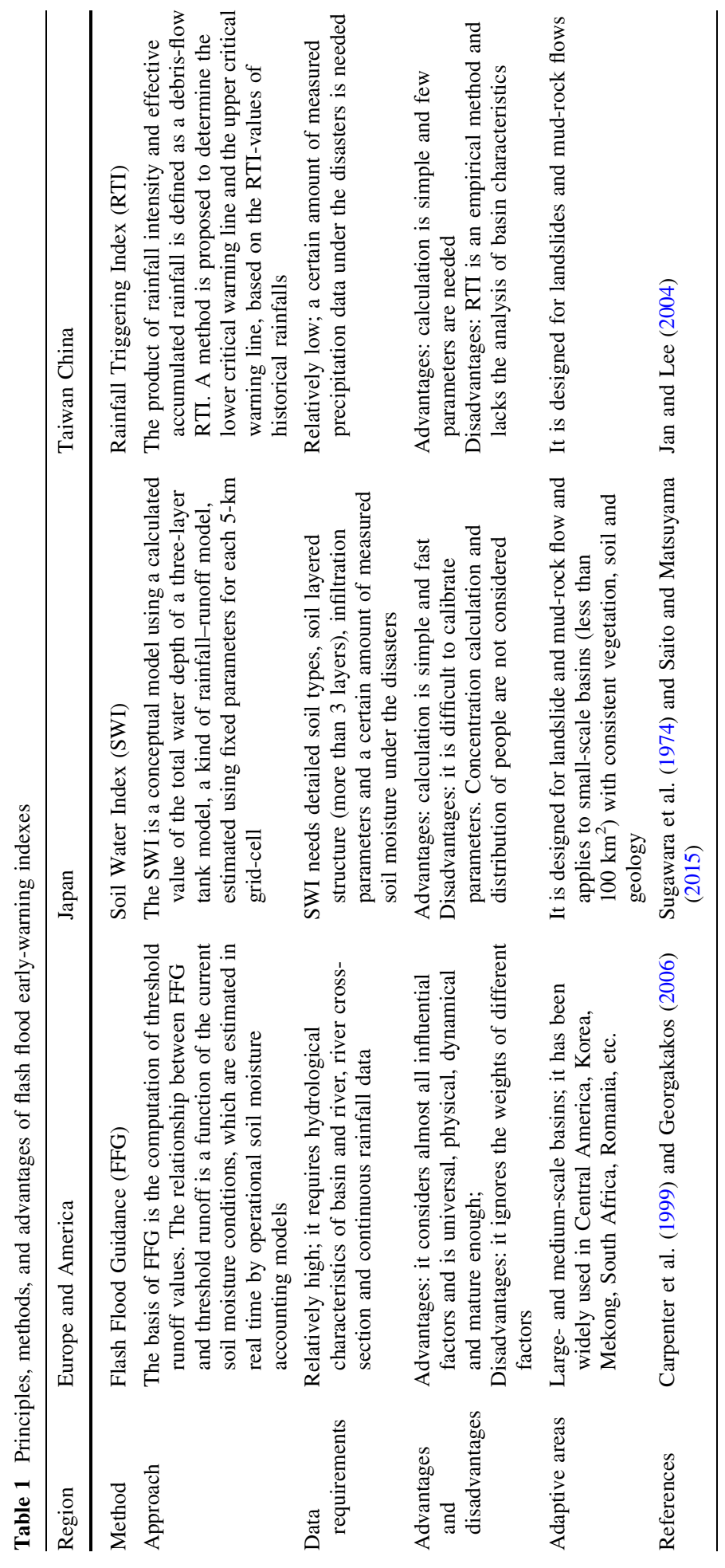




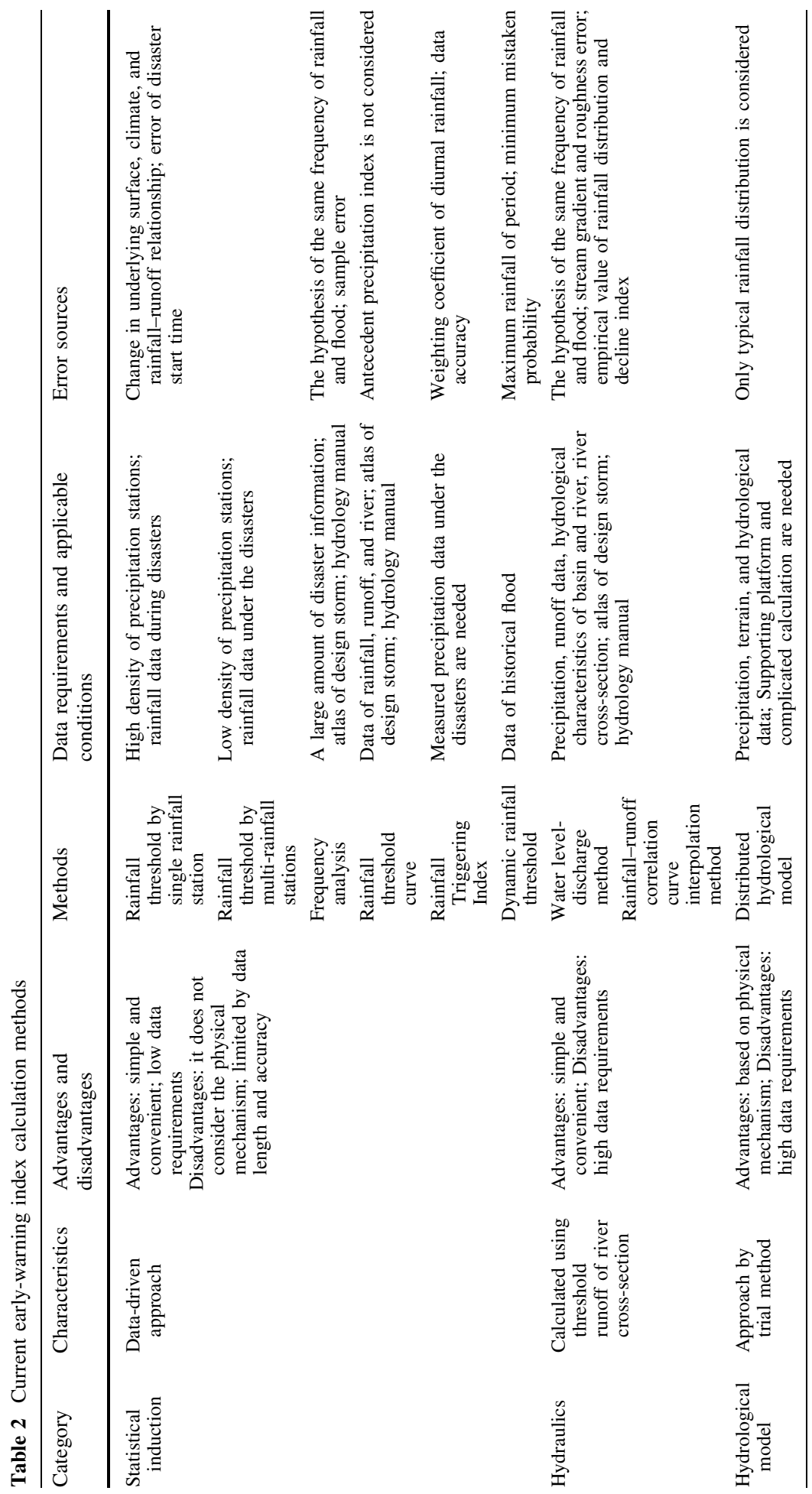


inhabitants of mountainous areas often reside by beaches and banks. Frequent construction activities and population growth also increase the vulnerability of humans to flash floods.

Flash flood disasters show spatial and temporal variabilities to a certain extent. China's flash flood disasters can be generally characterized as follows. Firstly, China's flash floods have a wide distribution and high frequency. Secondly, the sudden occurrence of flash floods in China leads to the high burstiness and difficulty in prevention. In general, disasters may occur just a few hours after the initiation of rainfall. Thirdly, China's flash floods are characterized by high destructiveness. Mountainous areas consist of rough terrain, steep slopes, and high river densities, and the population and property (estates, farmlands, etc.) are often distributed in flat lowlands. Therefore, flash floods can cause major disasters in a short period of time. Fourthly, China's flash floods have a strong seasonality. Flash floods are heavily concentrated in the period from May to September, especially from June to August. Lastly, China's flash floods have a distinct regional pattern, frequently occurring in Southwest China, Qin-Ba Mountain Areas, hilly areas in south Yangtze River, and southeastern coastal areas. Therefore, exploring flash flood earlywarning methods is of high importance.

\section{Methods for flash flood early warning in China}

\subsection{Methods and achievements in the first stage (2006-2012)}

During 2006-2010, China started the implementation of flash flood prevention, and gradually established nationwide county level flash flood monitoring and early-warning platforms in 2058 counties. Real-time monitoring of precipitation and water level could be achieved by establishing rainfall and gauging stations, and flash flood warnings could be issued through forecasting analysis and decision-making. Subsequently, preventive measures could be taken at the individual level. In the county level flash flood monitoring and early-warning platform, rainfall warning measures include preparation for evacuation and immediate evacuation. According to different forecast periods, pre-warning time is divided as: 1, 3, 6, and $24 \mathrm{~h}$. According to geographic features and historical data of precipitation and discharge, the precipitation-discharge relationship and parameters of hydrology have been summarized for each province in China. In the first stage, the main calculation methods of early-warning indexes were based on empirical methods. Empirical methods refer to methods in the Hydrology Handbook of each province, including atlas of design storm and flood for medium and small basins and simple theoretical derivation. Taking Henan Province as an example,

$$
\mathrm{Q}_{\mathrm{m}}=0.278 \varphi \frac{S}{\tau^{n}} F
$$

where $\mathrm{Q}_{\mathrm{m}}$ is peak discharge, $\varphi$ is runoff coefficient, $S$ is rainfall intensity, $\tau$ is concentration time, $F$ is drainage area, and $n$ is declining index (Henan Province Hydrology Bureau 1984). According to historical flood disasters, topography and landforms, storm characteristics, and engineering experience, engineers developed a flash flood earlywarning index. The early-warning index distribution map in the first stage is shown in Fig. 2. The resolutions of the early-warning index distribution maps are $1 \mathrm{~km} \times 1 \mathrm{~km}$. White areas were not included in the flash flood prevention system (such as flat areas), so data are not available. The distribution of the 1-h early-warning index is consistent with 1-h isohyet. However, compared to other provinces, the 6- and 24-h early-warning indexes 


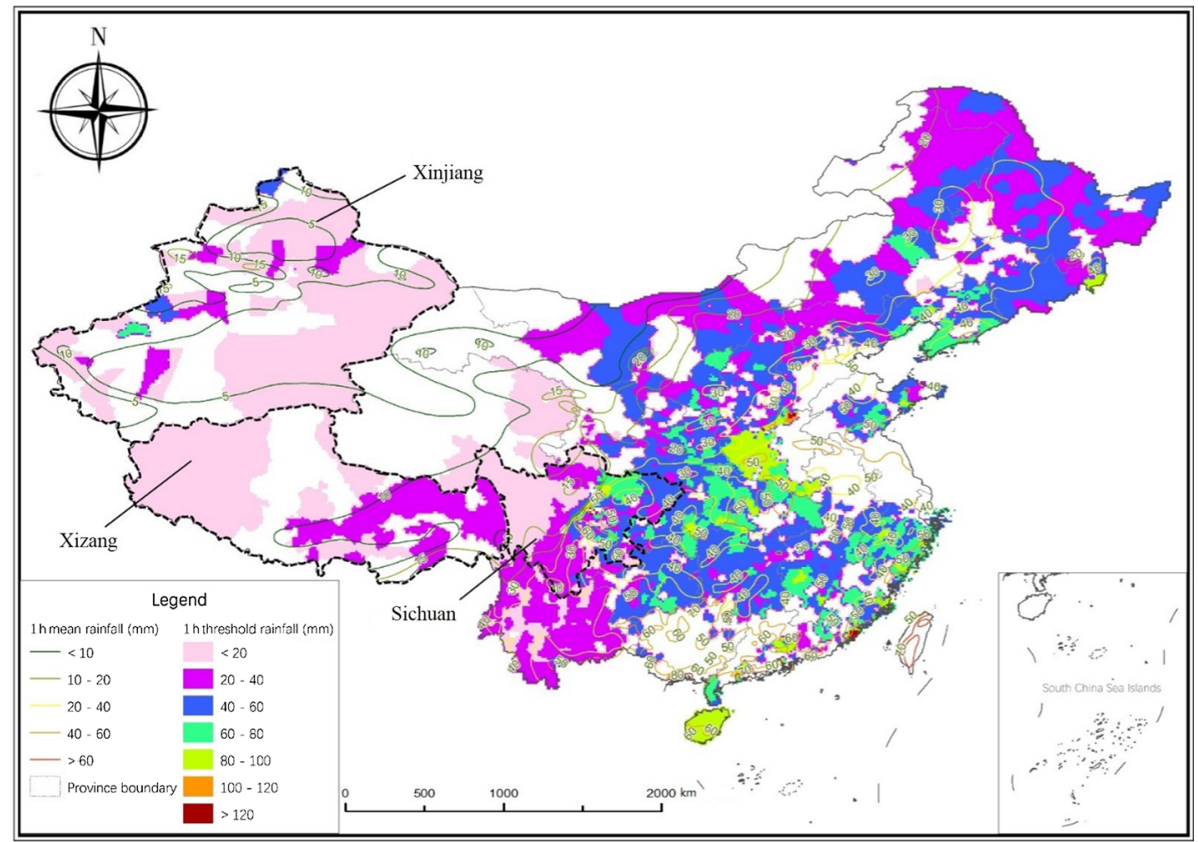

(a)

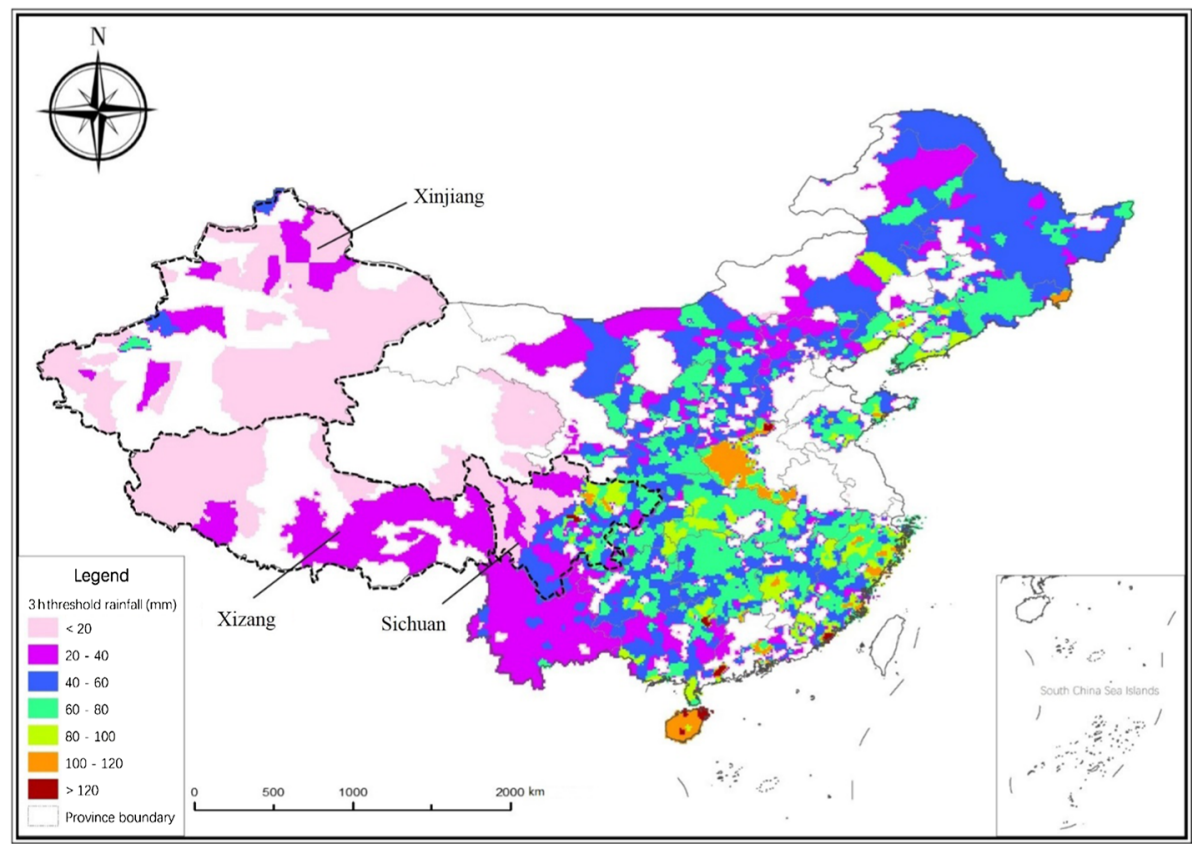

(b)

Fig. 2 Distribution map of the early-warning index in the first stage. a 1-h pre-warning time, b 3-h prewarning time, c 6-h pre-warning time, d 24-h pre-warning time 


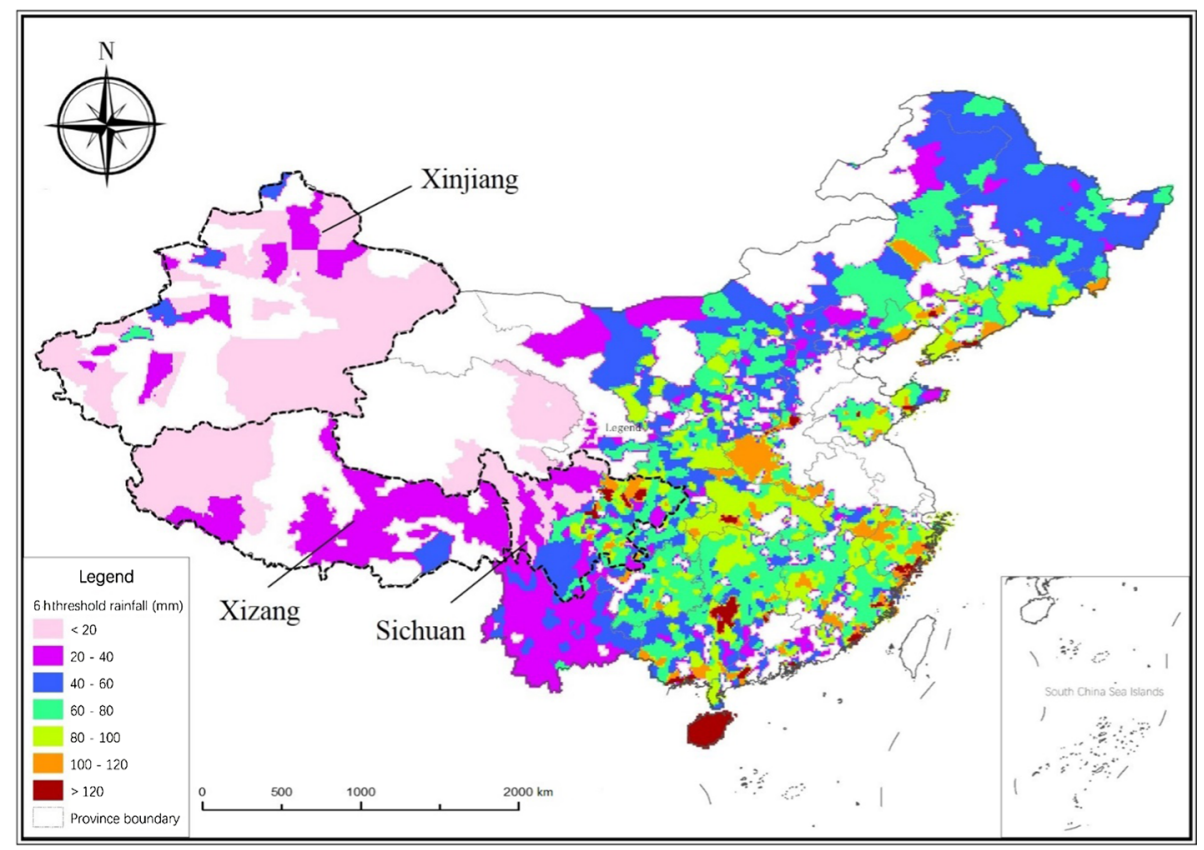

(c)

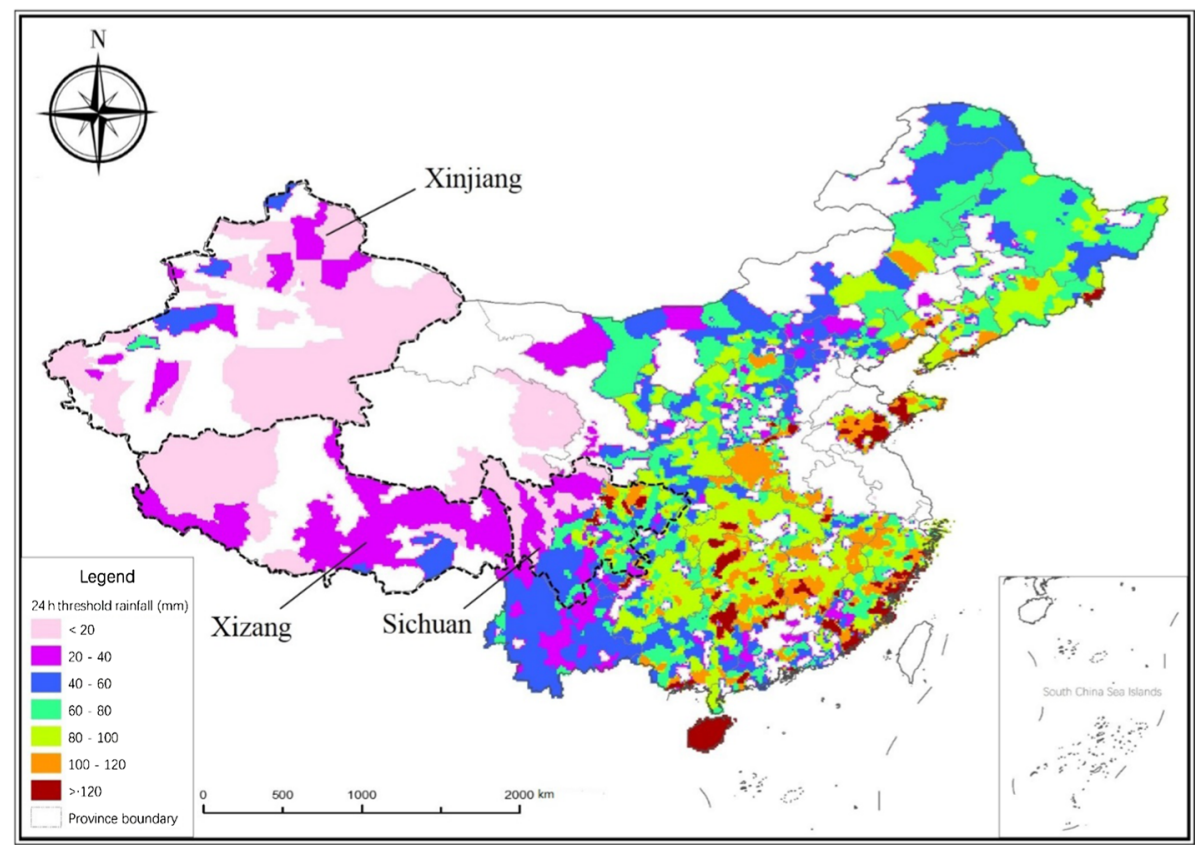

(d)

Fig. 2 continued 
of Xinjiang province, Xizang province, and Sichuan province (marked by black dotted lines) were lower under the same rainfall. The 24-h early-warning index is further divided into more levels, and its frequency was nearly 3 times that of the 1-h index. In the middle areas and southeastern coastal areas of China, the distribution of the 24-h early-warning index was more refined than that of the 1-h index. This means that the distribution of the 24-h index in these areas was much more asymmetrical. However, the current rainfall early-warning index is not accurate enough because it is determined on the basis of the empirical value, and storm characteristics, underlying surface condition, disaster prevention position, and inhabitant distribution are not sufficiently taken into account. In the first stage, the index was calculated mainly on the basis of historical flash flood disaster data, and therefore, large errors of forecasting and warning still remain.

\subsection{Methods and achievements in the second stage (2013-present)}

In May 2013, China's Ministry of Water Resources with the Ministry of Finance issued "National Flash Flood Prevention and Control Implementation Plan (2013-2015)," and then China moved into the second stage of flash flood prevention and control construction, including nationwide flash flood hazard investigation and evaluation, improvement and supplementation of non-structural measures, national middle-small rivers and dam reinforcement. The national flash flood hazard investigation and evaluation included flash flood prevention areas in 2058 counties. The general investigation involved basic information of village and small watersheds (such as geographic information and population information), hydro-meteorological data, water-related engineering, and so on (Li et al. 2014). Detailed investigation was conducted on the location and elevation of houses by rivers, inhabitant distribution, historical flood tracking and records, and cross and vertical sections of river channels, emphasizing on flash flood prevention in towns and villages. Satisfactory results were obtained in the analysis of the current flood-control ability and flood risk mapping in 150,000 villages near rivers in the prevention areas. To solve the problems and shortcomings faced in the first stage, nationwide flash flood hazard investigations were conducted to analyze in detail the location and elevation of houses near rivers, underlying surface characteristics, stream gradient and rainstorm behaviors, etc. The water leveldischarge method was used to calculate the largest cumulative rainfall in every period by rolling computation (Hu et al. 2015). By comparing the largest cumulative rainfall with the early-warning index, decisions are taken on whether to issue flash flood warnings. Only specific periods of threshold rainfall could be calculated by the water level-discharge method. This method is applied only to catchments in which long-term (more than 30 years) rainfall data of the outlet section are available. A flowchart for the calculation of the early-warning index is shown in Fig. 3. The threshold water level is determined by national flash flood hazard investigation instead of bank-full discharge. The value of threshold water level is related to the house with the lowest elevation in the village and underlying surface. Since 2013, the threshold water levels of all flash flood prevention areas have been obtained.

Compared with the calculation unit in the first stage, a transformation from county to village level could be realized in the second stage. In the main prevention areas, the index has been calculated for most villages nearby rivers. The area of catchments in this stage is relatively small and the concentration time is very short, so the 24-h early-warning index has become invalid. The distribution map (resolution: $1 \mathrm{~km} \times 1 \mathrm{~km}$ ) of the early-warning index in the second stage is shown in Fig. 4. White areas are not flash flood prevention areas (such as flat area), so data are unavailable. Compared with the distribution map of the 


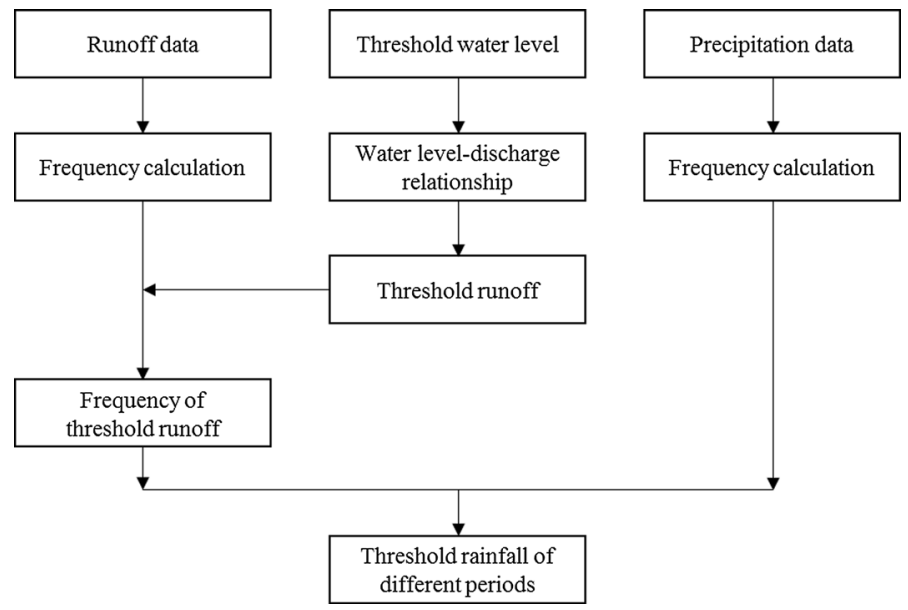

Fig. 3 Flowchart of the calculation of rainfall early-warning index

first stage, distribution is more detailed in the middle areas and southeastern coastal areas of China. However, because of the regional divergence, variations occur between the northeast region and Xinjiang province (marked by black dotted line). The water leveldischarge method has some disadvantages, such as ignoring antecedent rainfall, assuming the same frequency of precipitation and floods, and lacking physical mechanism, but this method does not require a special support platform and is convenient for local application.

\subsection{Early-warning index calculation methods for the next stage}

Since 2006, research on flash flood early-warning in China has gradually developed into scientific and accurate early-warning indexes from the initial stage where rainfall characteristics, underlying surface condition, and inhabitant distribution were not included. However, the dynamic variation of rainfall intensity and antecedent rainfall have not been fully considered; the description of the underlying surface is not accurate enough; and the flash flood early-warning index has remained static. Considering previous experiences and advanced technologies, the following indexes are proposed for the next stage: (1) real-time dynamic warning index based on automatic monitoring systems, monitoring and forecast platforms, and hydrological models; (2) compound warning index applicable to simple precipitation stations that record rainfall intensity, antecedent rainfall, and cumulative rainfall; and (3) meteorological warning index based on 24-h rainfall forecast, real-time information, and meteorological warning models. Furthermore, three warning modes based on these warning methods (warning platform, local warning, and meteorological warning) could improve the accuracy of China's flash flood monitoring and forecasting, and provide a reference or standard for upgrading the current warning index of each province.

(1) Real-time dynamic warning index

The main modules of this index include off-line threshold runoff, real-time soil moisture, and flash flood warning models. Data sources include data from the investigation and evaluation of flash flood hazards and multi-source rainfall data. Threshold runoff can be obtained through the investigation and evaluation (threshold water level, water level under a certain frequency, relationship between water level and runoff, and standardized unit 


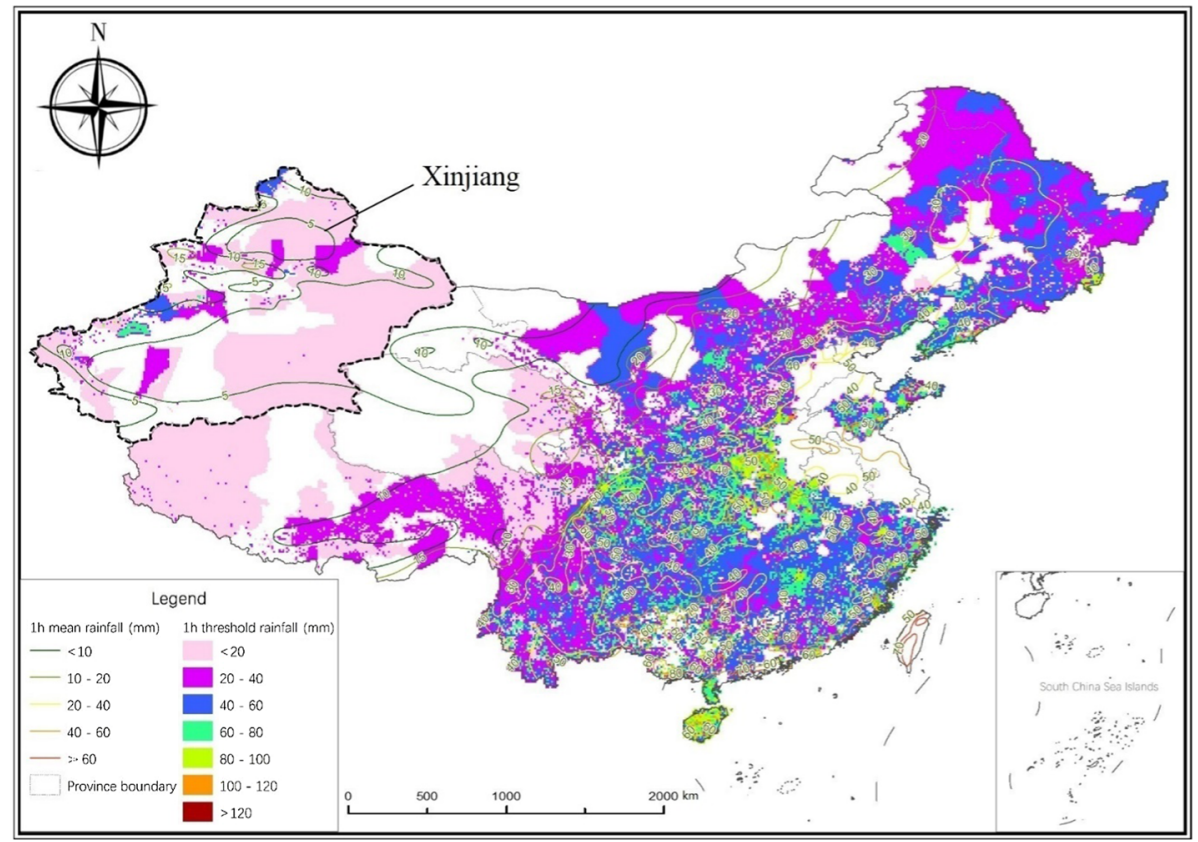

(a)

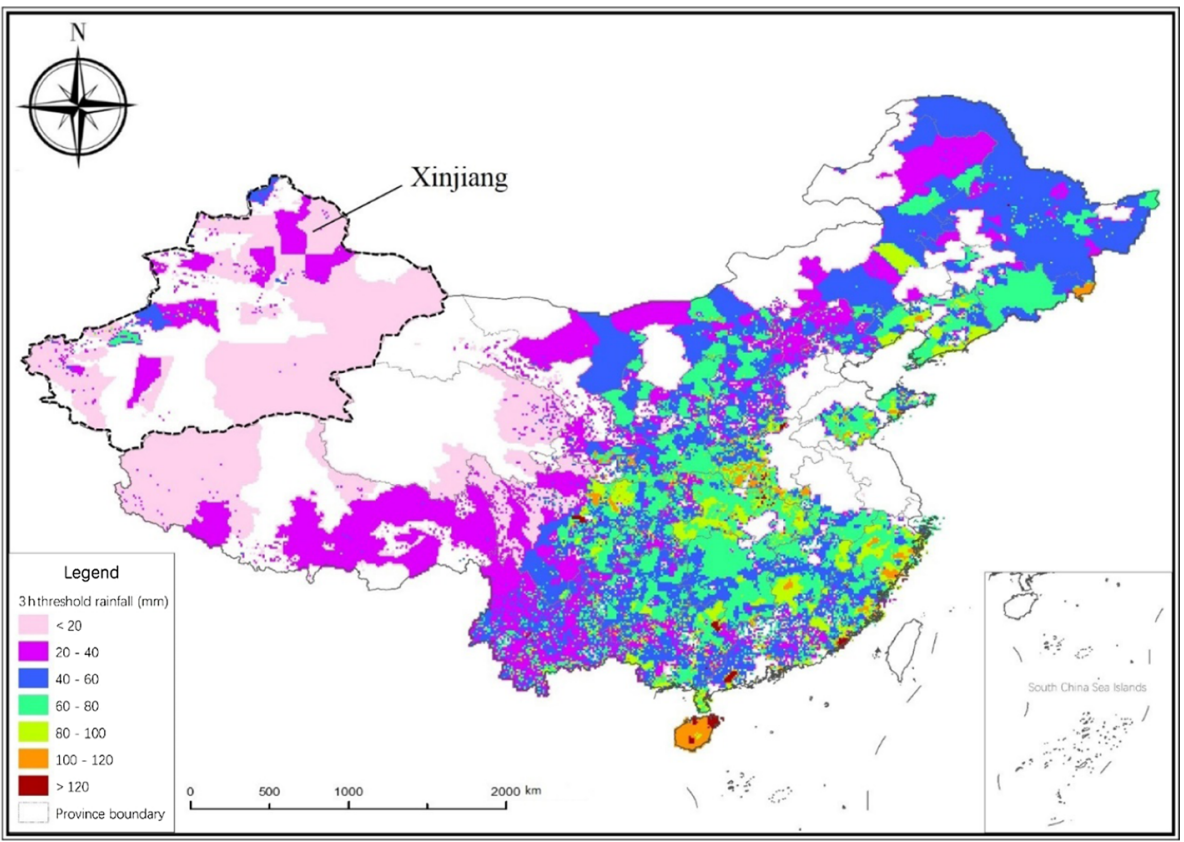

(b)

Fig. 4 Distribution map of the early-warning index in the second stage. a 1-h pre-warning time, b 3-h prewarning time, c 6-h pre-warning time 


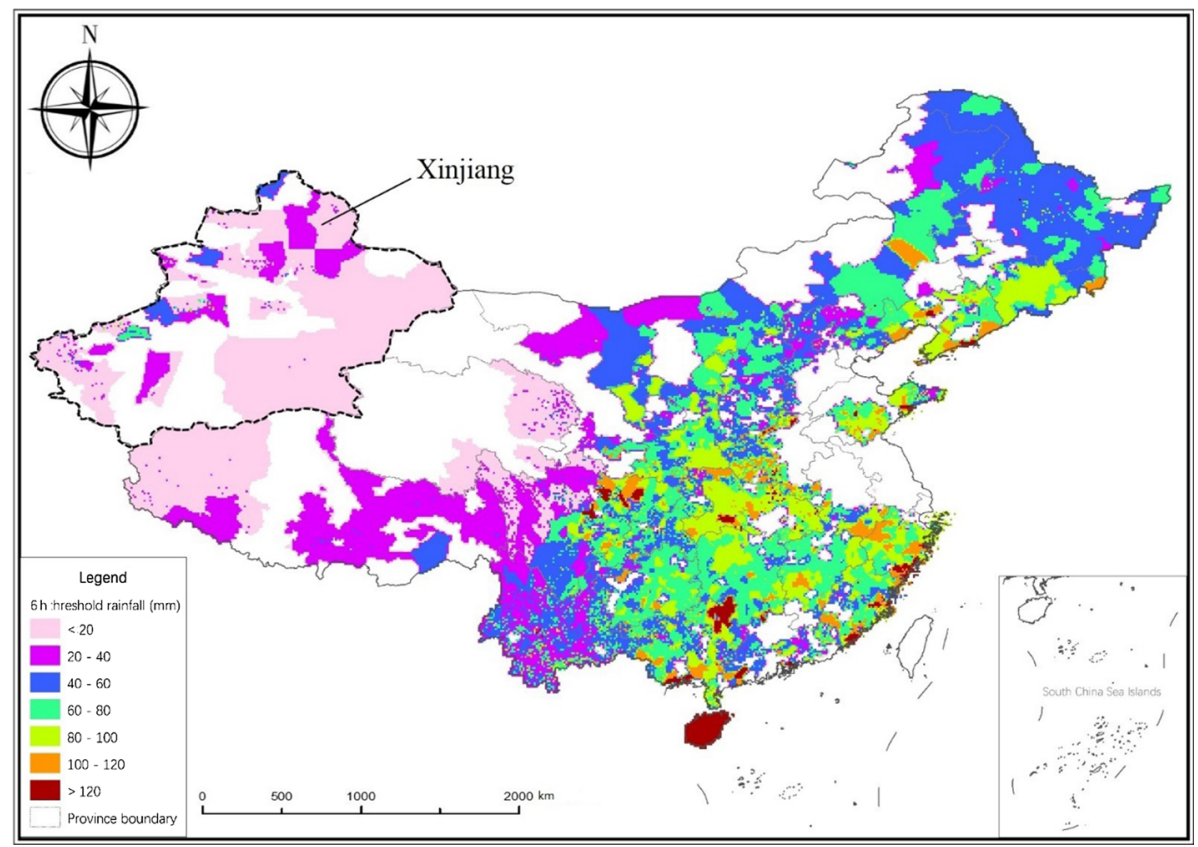

(c)

Fig. 4 continued

hydrograph) and distributed hydrological models. Soil moisture can be calculated using the antecedent precipitation index, modified SCS-CN, saturation excess runoff generation model, infiltration excess runoff generation model, mixed runoff generation model, and so on. In the flash flood warning model, the warning information is generated after t-hour warning index $(P c)$ is compared with t-hour rainfall $(P t)$ measured using multi-source data. A flow chart of the calculation of the real-time dynamic warning index is shown in Fig. 5. The data requirement is relatively high, demanding real-time rainfall data, underlying surface data, and historical runoff data. A special support platform is essential, and its calculation is relatively complicated. This method has strong and wide applicability because it takes into account local information. Decision makers can determine whether or not to issue flash flood warning after comparing threshold rainfall with multi-source rainfall data (rain gauge, radar, and weather forecast). The real-time dynamic warning index is of utmost importance in the development of the future flash flood warning system and has a potentially broad application in China.

(2) Compound warning index (CWI)

CWI is a method based on RTI (Jan and Lee 2004) under different probabilities of flash floods $(10,50$, and 90\%) and involves all major flash flood related factors (e.g., rainfall intensity, antecedent rainfall, and cumulative rainfall). At some point of time during a rainfall event, the value of compound warning index is determined by the product of rainfall intensity and effective cumulative rainfall, which is the sum of antecedent rainfall and cumulative rainfall. On the basis of rainfall data, CWI provides the upper and lower boundaries of rainfall warning. Warning area can be divided into three categories: low, medium, and high probabilities of flash floods. 


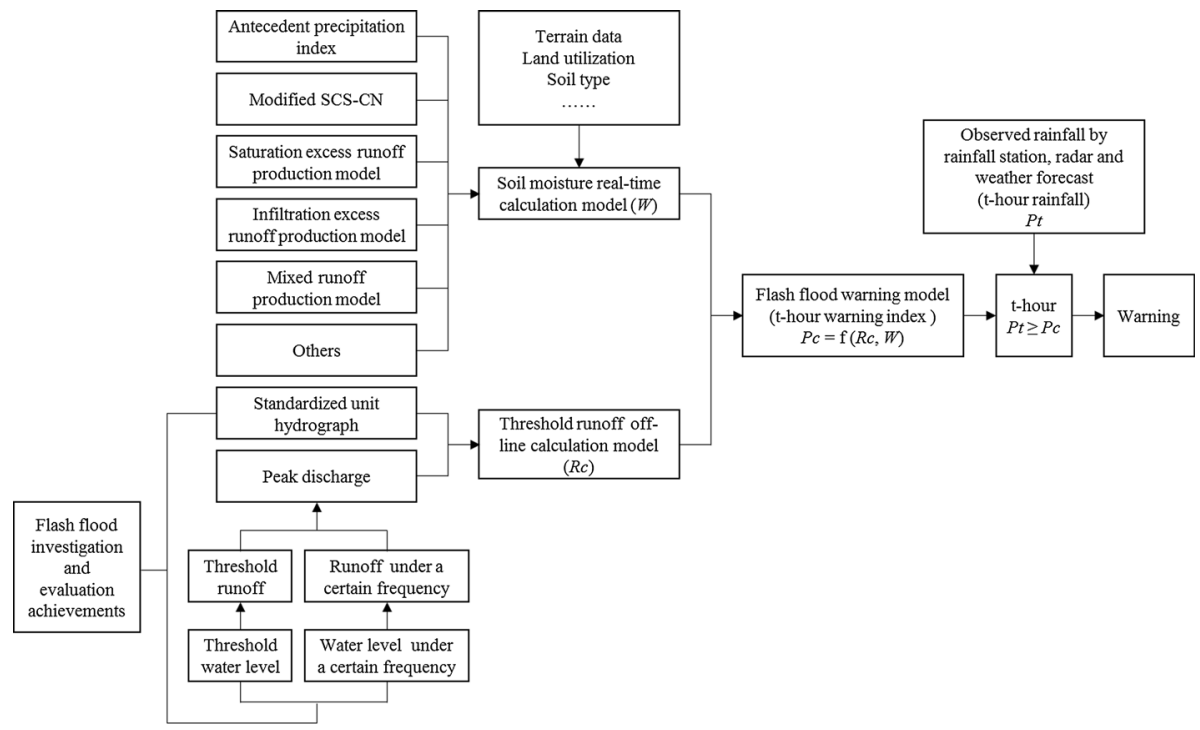

Fig. 5 Flowchart of the calculation of real-time dynamic warning index

CWI does not require any special support platform, and its objectives are straightforward. However, this method is only based on statistical analysis and does not consider hydrological factors (rainstorm behavior and characteristics of runoff). With further developments in the investigation and evaluation of flash flood hazards, updated rainfall data and data collection from ungauged regions would enable fundamental improvements of CWI.

\section{(3) Meteorological warning index}

Since July 2015, China's Ministry of Water Resources with the China Meteorological Administration has been developing the flash flood meteorological warning index. The meteorological warning index is calculated using a meteorological early-warning model by collecting 24-h rainfall forecasts and real-time information. The obtained flash flood earlywarning information is provided to the weather bureau and 3-6-h rainstorms are then forecasted. At present, Chinese scholars are attempting to combine the calculation of the index and the investigation and evaluation of flash flood hazards in order to improve the prediction grid resolution $\left(0.1^{\circ} \times 0.1^{\circ}\right)(\mathrm{Li}$ et al. 2007; Liao 2012; Qiu and Zi 2013; He and Li 2014; Chen and Li 2015; Zi et al. 2015).

The calculation and application of flash flood early-warning indexes are limited by the insufficient knowledge of storm runoff generation and its space-time distribution. At present, all the early-warning index calculation methods are hindered by the low accuracy of forecast rainfall. The flash flood early-warning system is still in the design stage and has not been verified. Therefore, a multi-source precipitation observation and fusion technology is urgently required. In the next stage, the accuracy of the flash flood early warning must be improved and the widespread implementation of the system must be realized through real-time rainfall observation using satellite and radar technology and distributed hydrological models. 


\section{Conclusions}

This study reviewed advances in China's flash flood early-warning system and reached the following major conclusions:

(1) FFG, which is widely used in Europe and the USA, is universal, physical, dynamical, and sufficiently robust. It is applicable primarily to large- and mediumscale basins. FFG has high potential as an important reference for the construction of the next stage of China's flash flood prevention platform at various levels (central, provincial, municipal, and county). SWI in Japan and RTI in Taiwan have been employed for debris flow; therefore, these methods may be applicable to flash flood prevention in Northeast and Northwest China.

(2) The development of methods for calculating flash flood early-warning indexes in China could be divided into three stages. In the initial stage, the empirical approach was used irrespective of physical mechanisms and its accuracy was relatively low. In the present stage, the investigation and evaluation of national flood hazards promoted the development of various early-warning methods. The water leveldischarge method is the main method, but it assumes that rainfall has the same frequency of flood and does not consider the effect of antecedent rainfall. In the next stage, national flash flood warning methods will be further improved and complemented. For this purpose, three indexes are proposed: real-time dynamic warning index, compound rainfall index, and meteorological warning index. These warning indexes are put forward in accordance with China's national situation and characteristics, and they may enable verification, calibration, and recheck of warning indexes at the provincial level. In short, China's flash flood prevention system is gradually becoming more scientific and achieving greater precision.

Acknowledgements This research was supported by the National Natural Science Foundation of China (Grant No. 51709033, 91547116), the projects of application of remote sensing on water and soil conservation in Beijing and its demonstration (Z161100001116102), the key technology on dynamic warning of flash flood in Henan Province (China) and its application (HNSW-SHZH-2015-06), the study on infiltration mechanisms of special underlying surface in coalmine goaf in Shanxi Province (China) and application of runoff generation and concentration theory (ZNGZ2015-008_2), and the research on spatio-temporal variable source runoff model and its mechanism (JZ0145B2017). We also would like to thank the handling editor as well as the anonymous reviewers for their constructive comments which considerably improved this paper.

Open Access This article is distributed under the terms of the Creative Commons Attribution 4.0 International License (http://creativecommons.org/licenses/by/4.0/), which permits unrestricted use, distribution, and reproduction in any medium, provided you give appropriate credit to the original author(s) and the source, provide a link to the Creative Commons license, and indicate if changes were made.

\section{References}

Carpenter TM, Sperfslage JA, Georgakakos KP, Sweeney T, Fread DL (1999) National threshold runoff estimation utilizing GIS in support of operational flash flood warning systems. J Hydrol 224:21-44

Chen C, Li J (2015) Comparative experiment on different combination of physics schemes of WRF model in mountain torrent early-warning. Yangtze River 46(7):60-63

Clark RA, Gourley JJ, Flamig ZL, Hong Y, Clark E (2014) CONUS-Wide evaluation of national weather service flash flood guidance products. Weather Forecast 29(2):377-392

Diakakis M (2011) A method for flood hazard mapping based on basin morphometry: application in two catchments in Greece. Nat Hazards 56:803-814 
Georgakakos KP (2006) Analytical results for operational flash flood guidance. J Hydrol 317:81-103

Golian S, Saghafian B, Maknoon R (2010) Derivation of probabilistic thresholds of spatially distributed rainfall for flood forecasting. Water Resour Manage 24:3547-3559

Gourley JJ, Erlingis JM, Hong Y, Wells EB (2012) Evaluation of tools used for monitoring and forecasting flash floods in the United States. Weather Forecast 27(1):158-173

Gourley JJ, Hong Y, Flamig ZL et al (2013) A unified flash flood database across the United States. Bull Am Meteorol Soc 94(6):799-805

Guo L, Zhang X, Liu R, Liu Y, Liu Q (2017) Achievements and preliminary analysis on china national flash flood disasters investigation and evaluation. Geo-Information Science (Accepted)

Han L, Xu Y, Pan G, Deng X, Hu C, Xu H, Shi H (2015) Changing properties of precipitation extremes in the urban areas, Yangtze River Delta, China, during 1957-2013. Nat Hazard 79:437-454

Hapuarachchi HAP, Wang QJ, Pagano TC (2011) A review of advances in flash flood forecasting. Hydrol Process 25:2771-2784

$\mathrm{He}$ B, Li Q (2014) Exploration on present situation and developing tendency of mountain flood disaster prevention technology. China Water Resour 18:11-13

Henan Province Hydrology Bureau (1984) Henan Hydrology Handbook 1-4 (in Chinese)

$\mathrm{Hu} \mathrm{H}$ (2016) Rainstorm flash flood risk assessment using genetic programming: a case study of risk zoning in Beijing. Nat Hazard 83:485-500

Hu Y, Yao X, Zhang C, Fang H, Fang G (2015) Investigation and assessment of flash flood effect and research on construction of early warning system: take Luxi reach of Chang River as a case. J China Hydrol 35(3):20-25

Huang P, Li Z, Chen J, Li Q, Yao C (2016) Event-based hydrological modeling for detecting dominant hydrological process and suitable model strategy for semi-arid catchments. J Hydrol 542:292-303

Jan CD, Lee MH (2004) A debris-flow rainfall-based warning model. J Chin Soil Water Conserv 35(3):275-285

Kim ES, Choi HI (2015) A method of flood severity assessment for predicting local flood hazards in small ungauged catchments. Nat Hazard 78:2017-2033

Kvočka D, Falconer RA, Bray M (2016) Flood hazard assessment for extreme flood events. Nat Hazard 84:1569-1599

Li J, Liao Y, Zhang B, Shen T (2007) The preliminary application of ensemble prediction in flash flood forecasting. Plateau Meteorol 26(4):854-861

Li C, Guo L, Liu C, Sun D (2014) Preliminary studies on mountain flood disaster evaluation. China Water Resour 18:14-17

Liao M (2012) Application of Doppler radar in early warning of mountain flood disasters. J Agric Catastrophol 2(4):84-88

Miao Q, Yang D, Yang H, Li Z (2016) Establishing a rainfall threshold for flash flood warnings in China's mountainous areas based on a distributed hydrological model. J Hydrol 541:371-386

Nieland C, Mushtaq S (2016) The effectiveness and need for flash flood warning systems in a regional inland city in Australia. Nat Hazard 80:153-171

Qiu H, Zi L (2013) Trail application of WRF model in early warning and forecast of mountain torrent disaster. Yangtze River 44(13):5-9

Saito H, Matsuyama H (2015) Probable hourly precipitation and soil water index for 50-yr recurrence interval over the Japanese Archipelago. SOLA 11:118-123

Sugawara M (1974) Tank model and its application to Bird Creek, Wollombi Brook, Bikin River, Kitsu River, Sanaga River and Nam Mune. Research Notes of the National Research Center for Disaster Prevention 11, pp 1-64

Sun D, Zhang D, Cheng X (2012) Framework of national non-structural measures for flash flood disaster prevention in China. Water 4:272-282

Wei Z, Shang Y, Zhao Y, Pan P, Jiang Y (2017) Rainfall threshold for initiation of channelized debris flows in a small catchment based on in-site measurement. Eng Geol 217:23-34

Yang T, Hwang G, Tsai C, Ho J (2016) Using rainfall thresholds and ensemble precipitation forecasts to issue and improve urban inundation alerts. Hydrol Earth Syst Sci 20:4731-4745

Yuan X, Liu Y, Huang Y (2017) Tian F (2014) An approach to quality validation of large-scale data from the Chinese Flash Flood Survey and Evaluation (CFFSE). Nat Hazard 2:1-12

Yucel I (2015) Assessment of a flash flood event using different precipitation datasets. Nat Hazard 79:1889-1911

Zeng Z, Tang G, Long D, Zeng C, Ma M, Hong Y, Xu H, Xu J (2016) A cascading flash flood guidance system: development and application in Yunnan Province, China. Nat Hazard 84:2071-2093

Zi L, Yang W, Yuan Y, Qiu H (2015) Experimental study of forecasting and early-warning technology for mountain torrent disasters based on rainfall threshold. Yangtze River 46(11):10-14 